\title{
Primary Histoplasmosis of Larynx Mimicking as Laryngeal Carcinoma
}

\author{
${ }^{1}$ Sampan S Bist, ${ }^{2}$ Shivanjali Sandhirr, ${ }^{3}$ Nadia Shirazi, ${ }^{4}$ Vinish Agrawal, ${ }^{5}$ Bhartendu Bharti
}

\begin{abstract}
Granulomatous diseases caused by infectious agents are being encounted more frequently nowadays. Infection by Histoplasma capsulatum is one such granulomatous disease. Isolated laryngeal histoplasmosis is a rare clinical entity. Because of its simulation with malignancy, it needs to be included in the differential diagnosis of neoplasm, both benign and malignant. We report an extremely rare case of isolated histoplasmosis, presenting as a primary infection of the larynx with no other systemic involvement in a 57 years old immunocompetent male. He presented with progressive hoarseness of voice, difficulty in breathing and mild dysphagia. Indirect laryngoscopy revealed multiple exophytic nodular lesions in endolaryngeal structures. The patient then underwent direct laryngoscopy biopsy under general anesthesia and the histopathological examination of the biopsy revealed chronic granulomatous inflammation, caused by histoplasma which was confirmed by using special Gomori's methanamine silver stain for fungi. Though it appeared like cancer clinically; biopsy confirmed the final diagnosis. The histopathologist must be informed about the possibility of histoplasmosis, because special dyes have to be used to confirm this diagnosis. The treatment is mainly medical with amphotericin B which remains the gold standard for treatment of this disease, although other drugs have also been used.
\end{abstract}

Keywords: Granulomatous, Histoplasmosis, Laryngeal malignancy.

How to cite this article: Bist SS, Sandhirr S, Shirazi N, AgrawalV, Bharti B. Primary Histoplasmosis of Larynx Mimicking as Laryngeal Carcinoma. Int J Phonosurg Laryngol 2015;5(1):28-31.

Source of support: Nil

Conflict of interest: None

\section{INTRODUCTION}

Histoplasmosis was first described by Darling in $1908 .{ }^{1}$ It is a systemic mycosis caused by Histoplasma capsulatum,

\footnotetext{
${ }^{1}$ Professor and Head, ${ }^{2}$ Junior Resident

${ }^{3}$ Associate Professor, ${ }^{4,5}$ Assistant Professor

1,2,4,5 Department of ENT, Himalayan Institute of Medical Sciences, SHRU University, Dehradun, Uttarakhand, India

${ }^{3}$ Department of Pathology, Himalayan Institute of Medical Sciences, SHRU University, Dehradun, Uttarakhand, India
}

Corresponding Author: Sampan S Bist, Professor and Head Department of ENT, Himalayan Institute of Medical Sciences SHRU University, Jolly Grant, Doiwala, Dehradun-248140 Uttarakhand, India, Phone: 01352471371, e-mail: sampanbist@ yahoo.com a dimorphic fungus that has been isolated from soil contaminated with bird or bat droppings in endemic areas, as reported in Brazil and central United States. ${ }^{2}$ It is a granulomatous fungal disease with variable clinical features. ${ }^{3}$ The course of histoplasmosis can be influenced by the immune status of the host and the quantity of infective propagules that the host is exposed too. ${ }^{2}$ Disease manifestations range from asymptomatic infection in normal host with low inoculum exposure to rapidly fatal disseminated infection in immunosuppressed host. This reflects the importances of cell mediated immunity against $H$. capsulatum. ${ }^{2}$ Upper aerodigestive lesions are chiefly associated with systemic disease, especially affecting patients with immunosuppression. Laryngeal lesions along with constitutional symptoms like dysphagia, loss of weight, loss of appetite, irregular low grade fever, often mimic a clinical diagnosis of squamous cell carcinoma or a granuloma. ${ }^{1}$ Two varieties of $H$. capsulatum are recognized: var capsulatum and var duboisii. They have different sizes, the capsulatum cells from 2 to $5 \mathrm{~mm}$ in diameter and duboisii from 10 to $15 \mathrm{~mm} .{ }^{4} \mathrm{H}$. capsulatum var capsulatum is endemic in United States and H. capsulatum var duboisii is endemic in West Central Africa. ${ }^{5}$ The incidence of laryngeal histoplasmosis is low when compared to the total number of cases. There are less than 100 publications on laryngeal histoplasmosis described in the medical literature since it was first described in 1940 by Brown et al. ${ }^{6}$ Isolated laryngeal involvement may lead to misdiagnosis and mistreatment as tuberculosis or malignancy, according to some reports. So, the former must be considered in the differential diagnosis of a patient with hoarseness of voice, and, moreover, in the differential diagnosis of tumors in the vocal folds, causing hoarseness. Biopsy must be limited, removing the least possible amount of tissue and the histopathologist must be informed of the possibility of using special dyes for diagnostic confirmation of histoplasmosis. We report a rare case of primary laryngeal histoplasmosis in a 57 years old immunocompetent male patient.

\section{CASE REPORT}

A 57 years old male presented with progressive hoarseness of voice, difficulty in breathing, and mild dysphagia of 4 months duration. He initially presented to a local 
specialist where a biopsy was performed that was consistent with chronic inflammation and no evidence of carcinoma. When his symptoms did not respond to therapy, he was referred to our tertiary care teaching hospital. The patient's medical history was not significant for any systemic illness. He was a chronic smoker with history of significant alcohol consumption. There was no history of loss of appetite or weight loss. He had spent the entirety of his life living in the Himalyan region of Uttarakhand in North India. Upon initial physical examination, the patient had notably hoarse voice with mild biphasic stridor. Flexible fiber-optic laryngoscopy revealed diffuse edema and inflamed endolaryngeal structures with multiple exophytic nodular lesions across the epiglottis, aryepiglottis folds and arytenoids. True vocal folds were obscured (Fig. 1). The hematological investigations and serum electrolytes were within normal limits. Serology tests (HbsAg, HIV and HCV) were negative. The patient was taken to the operating room for direct microlaryngoscopy with biopsy and the specimen was sent for histopathological examination. His larynx was noted to be friable, with a diffuse ulceronodular appearance. Histopathological examination (Figs 2A and B) of the tissue using hematoxylin-eosin stain revealed chronic granulomatous inflammation, caused by histoplasma. There was a diffuse infilterate of neutrophils, lymphocytes, and macrophages and few organisms. On oil immersion, scattered multiple organisms, of 2 to $5 \mathrm{~mm}$, within the macrophages with peripheral halo were seen on Gomori's methanamine stain, suggestive of histoplasma. The patient was managed conservatively on amphotericin B. A total of $86 \mathrm{mg}$ was given with periodic monitoring of serum electrolytes and creatinine. A pulseblood pressure charting was done during the whole infusion. No adverse effects were noted. The patient

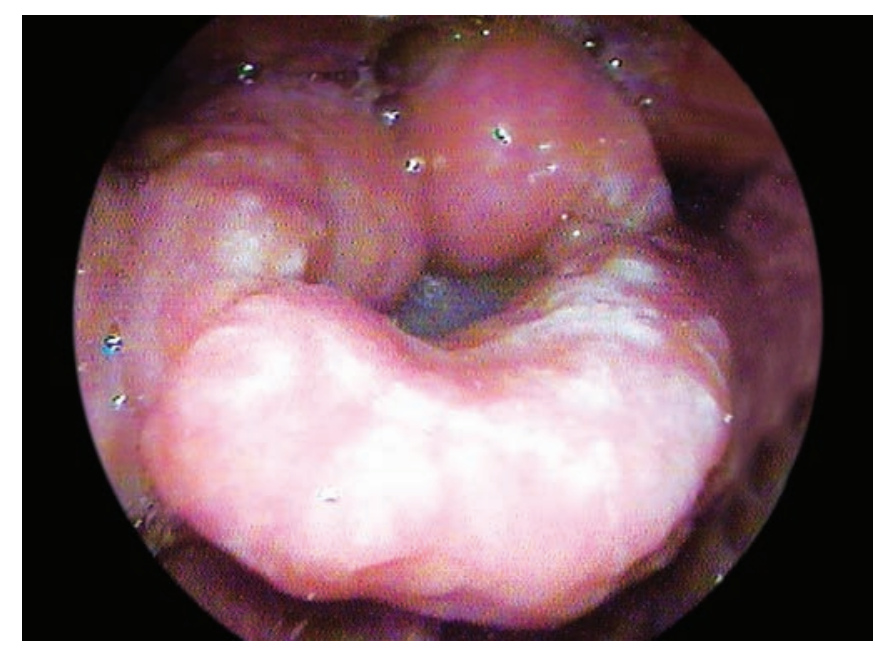

Fig. 1: Laryngoscopy showing exophytic nodular lesions in endolaryngeal structures was switched to a course of itraconazole $150 \mathrm{mg}$, twice a day for a period of 8 weeks and discharged on oral itraconazole. Near complete resolution of the lesions and relative return of normal vocal cord function was seen in 3 months. The patient on subsequent follow-up for a year showed healing of laryngeal lesions with complete resolution of symptoms and dramatic improvement in voice quality.

\section{DISCUSSION}

Histoplasmosis is the most common endemic mycosis in North America. Other endemic regions are parts of Africa, Australia, Eastern Asia, particularly India and Malaysia. ${ }^{4}$ Several cases have been reported from India since 1954, but in only some of them was the diagnosis confirmed on culture. From 1968 to 1992, 25 authentic cases from India have been reported. In 19 of them, the lesions were confined to oral cavity. Histoplasmosis is rarely reported from India, perhaps because of its varied presentation and lack of awareness among treating clinicians. Histoplasmosis
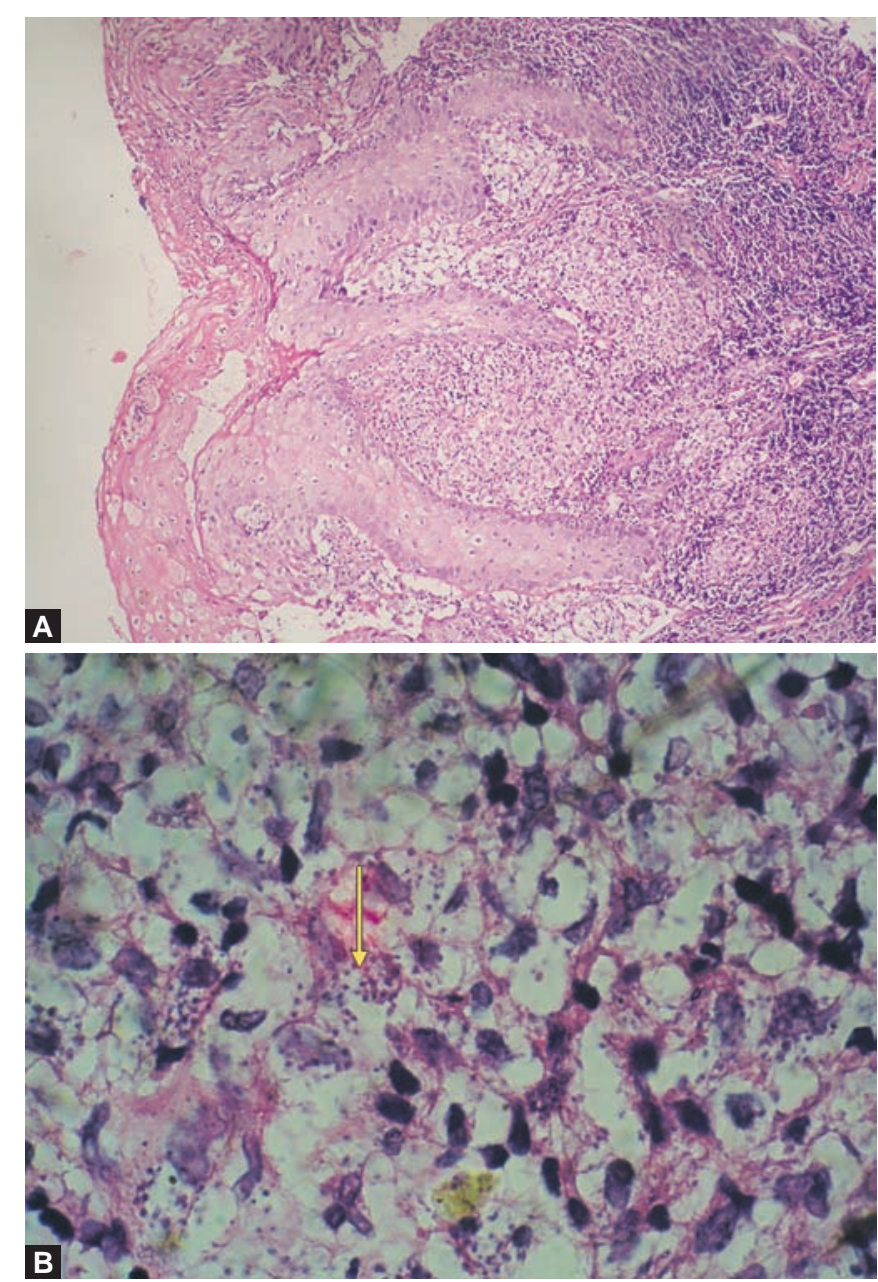

Figs 2A and B: (A) Microphotography ( $\mathrm{H}$ and E 10x) showing the sub epithelial stroma was densely infiltrated by inflammatory cells and (B) Microphotography ( $\mathrm{H}$ and $\mathrm{E} 40 \mathrm{x}$ ) showing the presence of numerous small capsulated rounded organisms 
is considered endemic in certain eastern states like West Bengal, where the skin positivity is $9.4 \%$ to histoplasmin antigen. ${ }^{3}$ Birds, bats and soil contaminated with their droppings are sources of infection. The spores are infective to humans. The disease is transmitted by the inhalation of airborne conidia. Infection can also follow the introduction of spores through skin and mucous membranes. As histoplasmosis has varied presentations, it is called as the syphilis of the fungal world. There are many known forms of histoplasmosis. The four main clinical variants are: acute pulmonary, acute disseminated, chronic disseminated and chronic pulmonary. Primary mucosal histoplasmosis is very rare. The primary lesion can occur on direct inoculation of the organism into mucosa. Primary lesion is a nodule or an indurated ulcer and there is local cervical lymphadenopathy with no systemic involvement. The primary mucosal lesions involutes spontaneously and do not usually occur in immunocompromised patients where disseminated histoplasmosis is more common. ${ }^{4}$ Our patient had no systemic involvement and was immunocompetent. In the chronic disseminated form of the disease, seen in immunocompetent adults, pulmonary symptoms are often less prominent and lesions of the skin and mucous membranes are more common, particularly oropharyngeal ulcerations. ${ }^{7}$ Laryngeal involvement occurs in the chronic disseminated form of histoplasmosis. Distinguishing laryngeal histoplasmosis from laryngeal carcinoma can be challenging, as in this case. Clinical suspicion and timely diagnosis of this disease are important in order to ensure that the patients receive appropriate treatment. Typical initial manifestations include hoarseness, dysphagia, and odynophagia, as well as fatigue and weight loss. ${ }^{8}$ Painful raised mucosal ulcerations may involve the oral cavity, tongue, pharynx, and larynx. Nodules appear as submucosal masses. ${ }^{9}$ When the only evidence of histoplasmosis is a mucosal lesion, investigations, such as chest radiography, sputum and urine cultures and bone marrow aspiration biopsy should be performed to look for disseminated disease. ${ }^{9}$ Laryngeal histoplasmosis is seldom diagnosed clinically. The differential diagnoses are carcinoma, tuberculosis, syphilis, lymphoma and other granulomatous disease of the head and neck. Microscopically, histoplasmosis may be confused with blastomycosis, tuberculosis and squamous cell carcinoma. Diagnosis is established by identifying the small intracellular yeast cells $(2-5 \mathrm{~mm})$ of histoplasma in smears of mucocutaneous lesions, sputum, peripheral blood, bone marrow, or in biopsy specimens. Leishmaniasis is the closest histoplathological differential diagnosis where the organisms are of similar size, but lack peripheral halo with nucleus and kinetoplast. Periodic Acid Schiff and Gomori's methanamine silver stains are positive only in case of histoplasmosis. Culture is the gold standard for the diagnosis of histoplasmosis, but its utility is limited by the waiting period of 2 to 4 weeks and decreased sensitivity. This may be due to the self limited nature of the disease. Organisms can be successfully isolated on Sabouraud's agar, although this process can take up to 6 weeks. ${ }^{10}$ Our patient underwent a biopsy and staining which confirmed histoplasma. Treatment for histoplasmosis has become considerably effective in recent years. Laryngeal histoplasmosis, as a form of disseminated infection, should initially be treated with amphotericin B for 1 to 2 weeks followed by itraconazole for severe cases, or only oral itraconazole for mild cases. ${ }^{11}$ Mucosal lesions typically respond within 6 to 8 weeks.9 Localized forms respond to oral itraconazole (200 mg once daily). ${ }^{3}$ Amphotericin B (accumulated doses of 500$1500 \mathrm{mg}$ ) is effective in disseminated histoplasmosis with itraconazole in follow-up period. ${ }^{5}$ Our patient received $86 \mathrm{mg}$ of amphotericin B and this was followed by oral itraconazole $150 \mathrm{mg}$, twice a day for a period of 8 weeks.

\section{CONCLUSION}

Histoplasmosis only rarely affects seronegative nonimmunocompromised individuals. In India, it is relatively endemic in eastern parts. Although laryngeal histoplasmosis is rare in North India, the practicing laryngologist should not be ignorant of its presentation. It should be considered as one of the differential diagnoses when patients present with signs and symptoms that resemble laryngeal carcinoma. The diagnosis can be confirmed by biopsy and histopathologist must be informed about the possibility of histoplasmosis, because special dyes have to be used to confirm this diagnosis. The lesion usually responds rapidly with 4 to 6 weeks of antifungal therapy.

\section{REFERENCES}

1. Rahman MT, Bakar NHA, Phillip R, Shamsudin AR. Oral histoplasmosis presenting as oral ulcer in Non-HIV patient. Southeast Asian J Trop Med Public Health 2004;35(2):389-390.

2. Antonello SV, Zaltron FV, Vial M, de Oliveira FM, Severo LC. Oropharyngeal histoplasmosis: report of eleven cases and review of litereature. Rev Soc Bras Med Trop 2011;44(1):26-29.

3. Bhagwat PV, Hanumanthayya K, Tophakhane RS, Rathod RM. Two unusual cases of histoplasmosis in human immunodeficiency virus infected individuals. Ind J Dermatol Venerol Leprol 2009;75(2):173-176.

4. Vijayan C, Suprakasan S, Kumar GN, Nair Ps, Jaypalan S. Primary mucocutaneous histoplasmosis presents as oral ulcer. Ind J Dermatol Venerol Leprol 2007;73(3):209.

5. Ferreira OG, Cardoso SV, Borges AS, Ferreira MS, Loyola AM. Oral histoplasmosis in Brazil. Pubmed. PMID: 12142871. Oral 
Surg Oral Med Oral Pathol Oral Radiol Endod 2002;93(6): 654-659.

6. Brown AC, Havens FZ, Magath TB. Histoplasmosis: report of case. Proc Staff Meet Mayo Clinic 1940;15(2):812-816.

7. Kauff man CA. Histoplasmosis. Essentials of Clinical Mycology. New York: Springer; 2011. p. 321.

8. Reibel JF, Jahrsdoerfer RA, Johns MM, Cantrell RW. Histoplasmosis of the larynx. Otolaryngol Head Neck Surg 1982;90(6):740-743.
9. Donegan JO, Wood MD. Histoplasmosis of the larynx. Laryngoscope 1984;94(2):206-209.

10. Pochini Sobrinho F, Della Negra M, Queiroz W, Ribeiro UJ, Bittencourt S, Klautau GB. Histoplasmosis of the larynx. Braz J Otorhinolaryngol 2007;73(6):857-861.

11. Wheat LJ, Freifeld AG, Kleiman MB, Baddley JW, McKinsey DS, Loyd JE, et al. Clinical practice guidelines for the management of patients with histoplasmosis: 2007 update by the Infectious Diseases Society of America. Clin Infect Dis 2007;45(7):807-825. 